\title{
Effects of Active Sting Damping on Common Research Model Data Quality
}

\author{
Michael J. Acheson* \\ Project Manager, NFMTC, NASA LaRC, Hampton Va., 23681, USA \\ S. Balakrishna ${ }^{\dagger}$ \\ Vigyan Inc., Hampton, Va., 23666, USA
}

\begin{abstract}
Recent tests using the Common Research Model (CRM) at the Langley National Transonic Facility (NTF) and the Ames 11-foot Transonic Wind Tunnel (11' TWT) produced large sets of data that have been used to examine the effects of active damping on transonic tunnel aerodynamic data quality. In particular, large statistically significant sets of repeat data demonstrate that the active damping system had no apparent effect on drag, lift and pitching moment repeatability during warm testing conditions, while simultaneously enabling aerodynamic data to be obtained post stall. A small set of cryogenic (high Reynolds number) repeat data was obtained at the NTF and again showed a negligible effect on data repeatability. However, due to a degradation of control power in the active damping system cryogenically, the ability to obtain test data post-stall was not achieved during cryogenic testing. Additionally, comparisons of data repeatability between NTF and 11-ft TWT CRM data led to further (warm) testing at the NTF which demonstrated that for a modest increase in data sampling time, a 2-3 factor improvement in drag, and pitching moment repeatability was readily achieved not related with the active damping system.
\end{abstract}

\section{Nomenclature}

$\bar{Y}_{j} \quad$ Jth nominal polar point y-value for all polar runs

$C_{D} \quad$ Drag coefficient stability axis

$C_{l} \quad$ Lift coefficient stability axis

$C_{m} \quad$ Pitching moment coefficient stability axis

$M \quad$ Total number of polar runs

$N \quad$ Total number of points per polar

Re Reynolds number

$s_{i} \quad$ Ith polar sample standard deviation

WOZ Wind off zero

$Y_{i, j} \quad$ Jth interpolated polar point y-value for ith polar run

Subscripts

$i \quad$ Repeat polar run index

j Polar point index

Symbols

$2 \sigma_{\text {mean }}$ Mean value of two times sample standard deviations (data repeatability)

$\alpha \quad$ Angle of Attack

$\delta Y_{i, j} \quad$ Y-data residual for the ith polar run and the jth polar point

\footnotetext{
*Project Manager, Technologies Application Branch, MS 237, NASA Langley Research Center, AIAA Member.

†Senior Research Scientist, Vigyan Inc.
} 


\section{Introduction}

$\mathrm{T}^{\mathrm{N}}$ today's global aerospace environment, ground test facility customers are requiring substantial improve1 ments in the accuracy and repeatability of test data. One only needs to examine industry articles on the Boeing versus Airbus design environment to understand some of the driving factors. Aircraft performance in terms of range, capacity and fuel consumption are vital to the success of these aircraft manufacturers. The need to significantly reduce aircraft development costs and time are critical to successful marketing of airframes to customers. In particular, the costs to airframe manufacturers for failure to meet quoted performance can be substantial.

One impediment that currently prevents ground test facilities from being able to consistently satisfy customer data quality goals is the impact of model dynamics on steady state test data. The dynamics problem of a test article and the associated support structure is ubiquitous to transonic ground testing. The effects of dynamics can increase data variation, as well as introduce bias in the final data. These effects vary among test articles, configurations and tunnel conditions and are well documented in the literature. ${ }^{1-3}$ Additionally, the safety implications of model dynamics are consistently one of the main drivers that prevent customers from achieving their desired data set. The need to develop methodologies to understand and/or mitigate model dynamics is greatly desired to begin to resolve these issues and ultimately achieve the highly accurate and repeatable ground test data for correlation to flight.

\section{Background}

$\mathrm{T}$ HE problems of test article dynamics typically arise out of compromises made in the force/moment measurement hardware and model support mechanism design. In the case of the force measurement hardware (balance), it is necessary to reduce the stiffness of the balance to allow for adequate strain such that sufficient force/moment measurement resolution is achieved. However, by reducing the stiffness of the balance, the balance (and associated model) dynamics become in general more "active." Similarly, the model support structure (sting) is typically reduced to the smallest (allowable) diameter that will minimize aerodynamic interference. Also, the support hardware length is increased to minimize aerodynamic interference from the attitude control mechanism. These requirements are in contrast with the need to maintain adequate strength for large aerodynamic loads and the stiffness necessary to minimize test article oscillation/divergence. Similar to the balance selection, smaller diameter/longer length support hardware tends to generate increased amplitude model dynamics.

The difficulties associated with model/support system dynamics have been known and studied for a long time $^{4-6}$ In fact, a large body of work has been done previously to minimize dynamics and to generate data correction methodologies. Techniques have been used in the past to predict apriori model/balance/support combinations that will exhibit extremely high dynamics. ${ }^{7}$ The main goal of these types of techniques is to influence the test article/structure design so as to prevent coalescence between mechanical modes, which can greatly inhibit testing. Data correction techniques exist which attempt to quantify the dynamics and apply appropriate physics based acceleration corrections to aerodynamic data. ${ }^{8-10}$ These techniques typically suffer from the difficult nature of accurately linking measured dynamics with data corrections. More generally, these techniques don't account for the fact that model dynamics can significantly alter aerodynamics (e.g. steady versus unsteady flow). In fact, if you had a highly accurate understanding of the dynamic aerodynamic flow characteristics such that very accurate corrections could be made to convert dynamic aerodynamic data to steady aerodynamic data, then it would not be necessary to conduct the test in the first place!

Many of the known methods to effect passive/active damping in order to mitigate model dynamics have been attempted at most transonic tunnels. The simplest methods tried have been to incorporate additional weights in the model to shift its associated natural frequencies away from those of the underlying support structure. At times, a simple exchange of the balance and/or sting support has greatly reduced the problems. On other occasions, the testing polars are simply modified to try to quickly "step through" environments that exhibit significant dynamics. Sometimes tunnel and support structure control methods have been modified to minimize the control impulses that may initiate test article dynamics. Other methods have used passive dampers built in the model support structure with some measure of success. Additionally, several variants of active damping systems have been tried. ${ }^{11,12}$ Typically, active damping systems utilize piezoelectric materials that are controlled to counteract observed system modes. The active damping methods (including the efforts the authors are currently undertaking) have met with significant success but still require significant 
improvement to consider the model/support structure vibration problem solved.

Complicating the problem is that the ground test facility and associated model support hardware dynamics significantly varies from facility to facility. This is readily apparent in the wide array of model support methodologies used in testing (arc sector, alpha/beta with heave, hydraulic actuators with pin joints and heave mechanisms, etc.) As a result, significant efforts have been attempted historically to minimize facility dynamics. However, these efforts typically involve significant expenses both in modifying the facility and then experimentally verifying the results. Unfortunately, due to the highly nonlinear interactions of specific test articles with the tunnel aeroelastically, the benefits of the modifications are very difficult to predict in advance.

The net result is that test article dynamics have effects on data significantly larger than customer desired repeatability goals. ${ }^{13,14}$ It is the desire of this research to further the state of the art of active damping by quantifying the effects that such systems have on aerodynamic coefficient data repeatability.

\section{Repeatability Results from the Common Research Model Tests}

The principle objective of the CRM tests (NTF Test 197, 11' TWT Test 11-0216) was to provide aerodynamic test facility data for validation of industry wide CFD predictions. ${ }^{15}$ A secondary objective of the test series was to design and utilize an active damping system which would enable test data to be obtained poststall for the generic CRM transport model. In particular, the CRM active damping system uses the NTF upper swept strut that was tested warm (NTF and 11' TWT) and cryogenically (NTF) in both the pre-stall and post-stall regions. The overall damper design and capability to enable post stall data acquisition during CRM testing is well documented by Balakrishna et al. ${ }^{16,17}$ The results detailed here are specifically targeted to validate that the use of an active damping system does not result in data quality deterioration within the normal transport industry testing region (pre-stall). Note that the effects of the active damping system on data quality (post-stall) are not examined in this work but are to be detailed in subsequent analysis.

Appendix B contains repeatability data for both the NTF CRM tests and the 11' TWT CRM test. These plots denote $\alpha$ versus the $2 \sigma_{\text {mean }}$ data repeatability (as described in Appendix A) for drag $\left(C_{D}\right)$, lift $\left(C_{L}\right)$ and pitching moment $\left(C_{M}\right)$. All of these stated coefficient quantities are given in the stability-axis frame of reference. The main results found during Tests 197,11-0216 from Appendix B are summarized in Table 1 for simplicity of analysis.

Some important observations on the repeatability results are noteworthy. First the repeatability of both facilities was observed to be quite good. In particular, for the wing/body $0^{\circ}$ tail configuration, Test 197 demonstrated drag coefficient repeatability of $\approx \frac{1}{2}$ count $(0.00005)$, while Test 11-0216 produced $\approx 1$ count (0.0001) at Mach 0.7 (See Figures 4,16) with the active damping system on. The other configuration of wing/body (no tails) resulted in repeatability for both Tests 197,11-0216 of $\approx 1$ drag count at Mach 0.7 (See Figures 10,22). It is the opinion of the authors' that the observed higher values i.e. reduced repeatability (similar for all three coefficients) between the two configurations at Mach 0.7 during Test 197 is consistent with the test observations that the "tailless" configuration had noticeably increased dynamics. Figure 25 shows that Test 210 produced better $2 \sigma_{\text {mean }}$ data repeatability over Test 197 at Mach 0.85 . This improvement was later shown to be due to increased sampling time. Note that Test 210 utilized only 7 points per polar while Test 197 utilized 13 point per polar which should be expected to produce an increase in the standard deviation of polars as calculated in Appendix A.

As observed in Table 1, the active damper state (on/off) had no noticeable impact on the coefficient repeatability (See Figures 4,16,5,10,22,11) for both configurations at Mach 0.7. A similar story is observed for the Mach 0.85 data where significantly worse (not active damping related) drag $2 \sigma_{\text {mean }}$ data repeatability is observed at both facilities with Test 197 yielding $\approx 3$ drag counts while Test 11-0216 produced $\approx 2$ counts (See Figures 6,17,7,12,23,13). Again, the Test 197 data at Mach 0.85 show no change in $0^{\circ}$ Tail configuration repeatability whether the damper was on or off (See Figures 6,7). However, a small (but measurable) improvement in the Test 197 Mach 0.85 (tailless) repeatability was observed with the active damping on as compared to the off case (See figures 13,12). However, further statistical analysis is required to enhance this observation. Note also that Table 1 shows within test repeatability for Test 197 but none for Test 11-0216. This is because no within test repeat data were acquired for the second CRM test (11-0216) due to insufficient testing resources.

While Test 197 was able to make direct observations on the effects of active damping state (i.e. repeats with damper off and on), Test 11-0216 was almost exclusively damper on. However, during the examination of 
Table 1. Aerodynamic coefficient repeatability results for Common Research Model testing: Re 8.06 million/ft, trips dots on.

\begin{tabular}{|c|c|c|c|c|c|c|c|c|}
\hline Test & Tail & $\begin{array}{c}\text { Active } \\
\text { Damper }\end{array}$ & Phase & Mach & $C_{D}$ & $C_{L}$ & $C_{M}$ & Figure \\
\hline 197 & On $^{*}$ & On & Within Series & 0.7 & $4.1133 \mathrm{E}-05$ & $1.1012 \mathrm{E}-03$ & $2.8565 \mathrm{E}-04$ & 4 \\
\hline $11-0216$ & On $^{*}$ & On & Within Series & 0.7 & $1.0782 \mathrm{E}-04$ & $2.0283 \mathrm{E}-03$ & $3.8204 \mathrm{E}-04$ & 16 \\
\hline 197 & On $^{*}$ & Off & Within Series & 0.7 & $4.1109 \mathrm{E}-05$ & $8.6884 \mathrm{E}-04$ & $3.1687 \mathrm{E}-04$ & 5 \\
\hline 210 & On $^{*}$ & Off & Within Series & 0.7 & $4.5562 \mathrm{E}-05^{+}$ & $1.1509 \mathrm{E}-03+$ & $2.3776 \mathrm{E}-04$ & 24 \\
\hline \hline 197 & On $^{*}$ & On & Within Series & 0.85 & $3.1119 \mathrm{E}-04$ & $3.0436 \mathrm{E}-03$ & $1.3367 \mathrm{E}-03$ & 6 \\
\hline $11-0216$ & On $^{*}$ & On & Within Series & 0.85 & $2.1895 \mathrm{E}-04$ & $2.1138 \mathrm{E}-03$ & $8.4273 \mathrm{E}-04$ & 17 \\
\hline 197 & On $^{*}$ & Off & Within Series & 0.85 & $3.1319 \mathrm{E}-04$ & $3.3571 \mathrm{E}-03$ & $1.3637 \mathrm{E}-03$ & 7 \\
\hline 210 & On $^{*}$ & Off & Within Series & 0.85 & $1.4779 \mathrm{E}-04+$ & $2.0950 \mathrm{E}-03+$ & $8.4431 \mathrm{E}-04$ & 25 \\
\hline \hline 197 & On $^{*}$ & On & Within Test & 0.7 & $4.3760 \mathrm{E}-05$ & $1.8551 \mathrm{E}-03$ & $5.6558 \mathrm{E}-04$ & 8 \\
\hline 197 & On $^{*}$ & On & Within Test & 0.85 & $3.3624 \mathrm{E}-04$ & $3.2186 \mathrm{E}-03$ & $2.7066 \mathrm{E}-03$ & 9 \\
\hline \hline 197 & Off $^{\dagger}$ & On & Within Series & 0.7 & $8.9766 \mathrm{E}-05$ & $1.2379 \mathrm{E}-03$ & $1.8115 \mathrm{E}-04$ & 10 \\
\hline $11-0216$ & Off $^{\dagger}$ & On & Within Series & 0.7 & $7.8534 \mathrm{E}-05$ & $8.9995 \mathrm{E}-04$ & $3.4971 \mathrm{E}-04$ & 22 \\
\hline 197 & Off $^{\dagger}$ & Off & Within Series & 0.7 & $9.2379 \mathrm{E}-05$ & $1.0791 \mathrm{E}-03$ & $1.8087 \mathrm{E}-04$ & 11 \\
\hline \hline 197 & Off $^{\dagger}$ & On & Within Series & 0.85 & $3.2720 \mathrm{E}-04$ & $2.5185 \mathrm{E}-03$ & $1.0674 \mathrm{E}-03$ & 12 \\
\hline $11-0216$ & Off $^{\dagger}$ & On & Within Series & 0.85 & $2.2672 \mathrm{E}-04$ & $1.8792 \mathrm{E}-03$ & $4.2014 \mathrm{E}-04$ & 23 \\
\hline 197 & Off $^{\dagger}$ & Off & Within Series & 0.85 & $3.6332 \mathrm{E}-04$ & $2.9349 \mathrm{E}-03$ & $1.1704 \mathrm{E}-03$ & 13 \\
\hline \hline 197 & Off $^{\dagger}$ & On & Within Test & 0.7 & $1.3246 \mathrm{E}-04$ & $1.4155 \mathrm{E}-03$ & $1.8607 \mathrm{E}-03$ & 14 \\
\hline 197 & Off $^{\dagger}$ & On & Within Test & 0.85 & $3.4072 \mathrm{E}-04$ & $2.8437 \mathrm{E}-03$ & $2.8553 \mathrm{E}-03$ & 15 \\
\hline \hline
\end{tabular}

${ }^{*}$ Configuration wing/body $0^{\circ}$ tails

† Configuration wing/body

+ Test 210 utilized only 7 points per polar while tests 197,11-0216 used 13

conditional sampling (described later) a small repeat set (2 runs each) of active damper off data was obtained for the $0^{\circ}$ Tail configuration (inverted) for both Mach 0.7 and 0.85 . The results (See Figures 22,23,18,20 ) imply no significant active damping effect on aerodynamic coefficient repeatability. As was noted earlier, Test 11-0216 exhibited superior repeatability performance (e.g. drag improvement $\approx 1$ count) over Test 197 at Mach 0.85. Since the 11' TWT typically utilizes Mach number conditional sampling whereas the NTF does not, the authors saw the need to quantify the effects of conditional sampling observed during Test 11-0216. Table 2 shows the results of conditional sampling for the $0^{\circ}$ Tails configuration at both Mach 0.7 and Mach 0.85 . Interestingly, at Mach 0.7 worse repeatability was observed while at Mach 0.85 repeatability was generally improved (except for $C_{L}$ ).

This heuristic conditional sampling analysis led the NTF team to investigate the possibility of implementation of conditional sampling at their facility. This work resulted in a subsequent CRM test, the NTF Test 210 Data Sampling Evaluation. This test was structured so as to provide the data needed to enable analysis of (irrespective of active damping): conditional sampling, effects of data sampling rate and period, effects of wind off zeros, and the effects of various data filtering methodologies. While the analysis of Test 210 data is not complete, some interesting observations to date have been made as shown in Figures 1,2.

Figure 1 shows repeatability results using various sampling time increments (2 to 15 seconds) from Test 210. Drag and pitching moment coefficients show exponential improvements in repeatability with sample time. In particular, an increase in sampling time from 2 seconds to 6 seconds improves drag coefficient repeatability from $\approx 3$ counts to $\approx 2$ counts at Mach 0.85 which implies that conditional sampling could provide future repeatability performance improvements at the NTF. Figure 2 is a representative plot of the wind-off zero (WOZ) study conducted during Test 210. This study was designed to determine the impact that utilizing periodic WOZs would have on data quality. The study was structured such that approximately 
Table 2. Effects of conditional sampling on aerodynamic coefficient repeatability results: Re $5.0 \mathrm{mil} / \mathrm{chord}$, trips dots on, active damping off, model inverted.

\begin{tabular}{|c|c|c|c|c|c|c|c|c|}
\hline Test & Tail & $\begin{array}{c}\text { Conditional } \\
\text { Sampling }\end{array}$ & Phase & Mach & $C_{D}$ & $C_{L}$ & $C_{M}$ & Figure \\
\hline $11-0216$ & On $^{*}$ & On & Within Series & 0.7 & $1.4641 \mathrm{E}-04$ & $1.5540 \mathrm{E}-03$ & $3.5048 \mathrm{E}-04$ & 18 \\
\hline $11-0216$ & On $^{*}$ & Off & Within Series & 0.7 & $1.0353 \mathrm{E}-04$ & $8.4988 \mathrm{E}-04$ & $1.9546 \mathrm{E}-04$ & 19 \\
\hline $11-0216$ & On $^{*}$ & On & Within Series & 0.85 & $2.4091 \mathrm{E}-04$ & $2.3030 \mathrm{E}-03$ & $8.7749 \mathrm{E}-04$ & 20 \\
\hline $11-0216$ & On $^{*}$ & Off & Within Series & 0.85 & $4.2804 \mathrm{E}-04$ & $1.5242 \mathrm{E}-03$ & $1.5270 \mathrm{E}-03$ & 21 \\
\hline
\end{tabular}

${ }^{*}$ Configuration wing/body $0^{\circ}$ tails

every 15 minutes a series of WOZs were taken followed by several repeat runs at various Mach numbers. This process was continued for a couple of hours. The WOZ acquisition process was modified to incorporate multiple $\alpha$ 's to enable linear regression, which was then used to quantify data acquisition system drift over time. The findings showed that the data acquisition system was extremely stable, yet the effects of utilizing the various WOZs was non-trivial $\left(\approx \pm \frac{1}{4}\right.$ drag count). Figure 2 shows the repeatability for one particular data run which utilized eight different WOZs in data reduction prior to computing repeatability. So in essence the repeatability of one data run is being compared to itself using a large set of different WOZs. The choice of this particular run was arbitrary as all runs in the study showed very similar trends. The results show as much as $\frac{1}{2}$ drag count differential for the same data run based on which WOZ was used. In addition, a residual repeatability sensitivity analysis was performed that demonstrated that small variations in WOZ balance readings reflected as slope changes in the residual plot lines of Fig. 2, while small variatons in WOZ angle readings resulted in small vertical bias in the residual plots lines. Further analysis is ongoing, however this study has demonstrated one of the primary limitations on within-test and test-to-test repeatability comparisons may be wind off zero related.

\section{Conclusions}

A systematic study utilizing the Common Research Model across two different transonic ground test facilities shows that the use of an active damping system has no detrimental effects on pre-stall aerodynamic coefficient data quality and may potentially provide modest data quality improvements during high dynamic segments of the test envelope. Further statistical analysis is warranted during both the pre-stall and post-stall testing envelopes to more definitively quantify active damping performance. However, this research makes a good case for widespread implementation of active damping in transonic testing since active damping does not negatively affect aerodynamic coefficient data, while it has demonstrated the ability to increase the aircraft performance testing envelope. It should be noted that the active damping system control laws utilized were designed to target the large amplitude dynamics associated with post-stall testing, so a potential area of future research would be to target damper performance towards pre-stall (smaller amplitude) dynamics and determine if any significant benefits are achievable thru modifications to control philosophy.

This research has also demonstrated typical aerodynamic coefficient repeatability performance that can be expected during transport model testing at both the National Transonic Facility and the 11-Foot Transonic Wind Tunnel utilizing a well documented methodology to compute repeatability. Clearly, the establishment of an "industry standard" method of computing data repeatability is desirable.

While not the focus of this research, a cursory examination of the impact of conditional sampling on data quality has been introduced. This analysis has demonstrated the need for further analysis and documentation as to the best methods to maximize conditional sampling performance both at 11' TWT and during the potential implementation at the NTF. The NTF Test 210, Data Sampling Evaluation has shown that significant improvements in data quality are readily achievable through sampling time. Future evaluation of this tests data will focus on potential implementation of conditional sampling at the NTF, methods to mitigate the effects of wind off zeros on data repeatability and a systematic analysis of the effects of data acquisition techniques (including filtering) on data quality. 


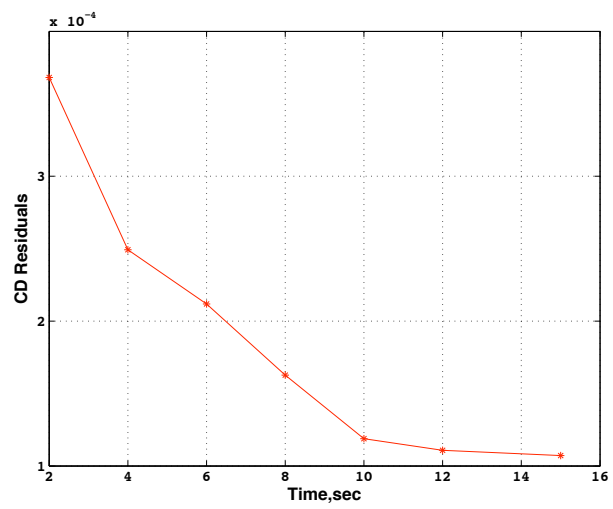

(a) $C_{D}$

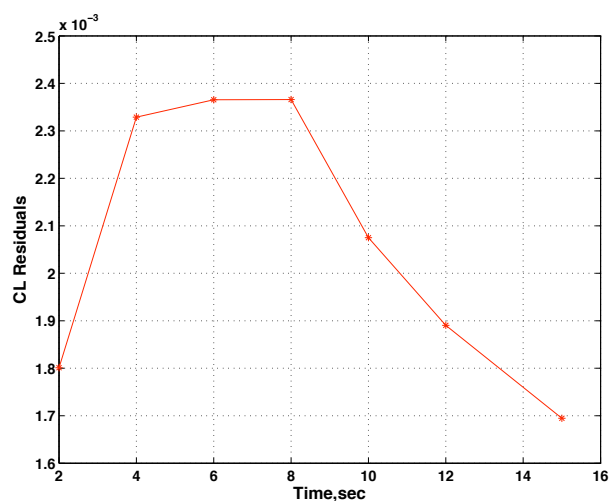

(b) $C_{L}$

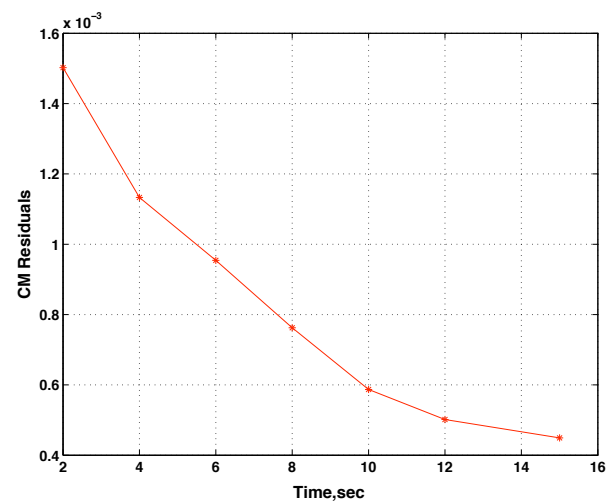

(c) $C_{M}$

Figure 1. Effects of sampling time on aerodynamic coefficient repeatability during NTF test 210: Mach 0.85 , Re 8.06 million/foot, $120^{\circ} \mathrm{F}$, config: wing/body $0^{\circ}$ tails, trip dots on, active damping off, within series repeatability.
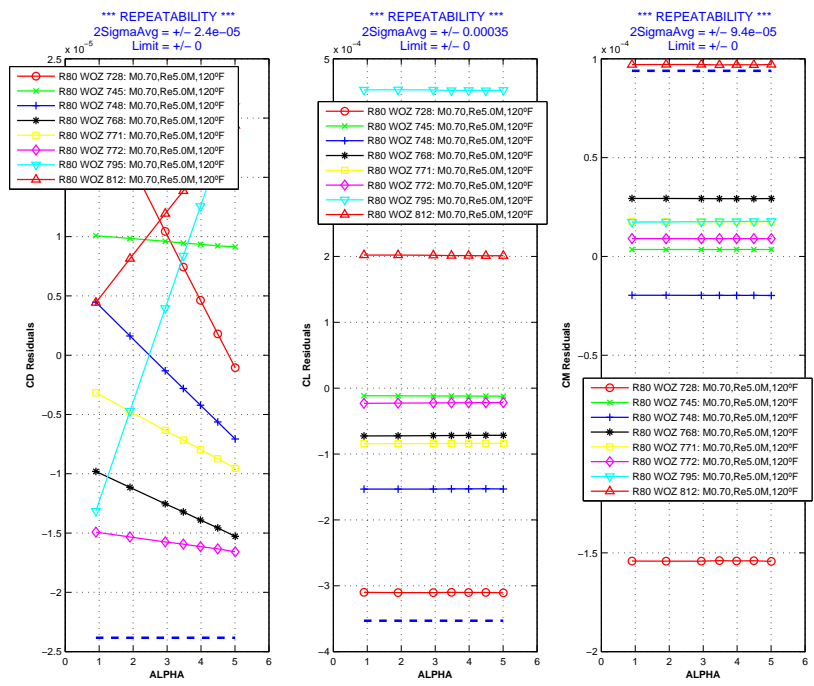

Figure 2. Sample results of WOZ study during NTF test 210: Mach 0.7, Re 8.06 million/foot, $120^{\circ}$ F, config: wing/body $0^{\circ}$ tails, trip dots on, active damping off. 


\section{Appendix}

\section{A. Data Repeatability Methodology}

The authors would like to emphasize the importance of detailing the exact methodology used to compute data repeatability in this paper since this paper deals not only with the sensitive topic of tunnel-to-tunnel comparisons, but also is attempting to validate the performance of new testing technology (i.e. active damping). Additionally, during literature searches, data repeatability is sometimes quoted but without full data repeatability methodology provided. ${ }^{18}$ For these reasons, the methodology, as well as step-by-step computations for an actual data example have been provided to ensure complete disclosure on the method utilized to definitively quantify the effects of active damping on data quality. It is interesting to note that having participated in testing at other wind tunnels (including during the tests described in this paper), there is no consensus agreement on a common standard for computing data repeatability.

The described methodology utilized in this paper is by no means the "best" one with which to compute data repeatability. In actuality, the methodology used in this analysis is based on one that is found in legacy NTF data plotting/analysis software. However, it is a straightforward method that when used within some basic guidelines yields suitable results. This legacy method utilizes linear interpolation (with extrapolation) of each repeat run to determine residuals and the associated $2 \sigma_{\text {mean }}$ sampled standard deviations. As noted by Wahls (et al), ${ }^{19}$ other methodologies based on polynomial fits are sometimes used. Another common way of describing data repeatability is to choose a "reference" run based on a set of repeats and to plot the difference of the remaining repeat runs relative to the selected reference run. This method gives some measure of the associated data scatter but results are determined on the basis of the chosen reference run.

One of the multivariate curve fitting methods detailed ${ }^{19}$ involves treating each data run separately and performing a least squares fit (independent variable $\mathrm{x}$ with dependent variable $\mathrm{y}$ ) fit to determine the polynomial coefficients (of the desired polynomial order). Each runs' associated polynomial is then evaluated at a standard set of independent variable values and the associated standard deviation can be computed. The NTF method utilized is similar to this method where linear interpolation is used to compute the standard deviation for each run separately and subsequently averaged to yield the quoted repeatability. Another multivariate method detailed, ${ }^{19}$ involves performing a polynomial fit (of desired polynomial order) for the complete set of run data. The result is a computed reference polynomial that "best" fits all observed data simultaneously. This computed reference polynomial is then used to assess the scatter of the observed data around the desired reference points.

The above multivariate polynomial methods provide a least squares regression fit of the data unlike linear interpolation methods, however these polynomial methods require determination of the polynomial order. Additionally, both polynomial multivariate methods described above suffer from the limitation that dependent/independent variables must be selected and that the regression performed is then one-dimensional. There is certainly a case to be made that some aerodynamic data could be treated as two variables where the magnitudes of scatter for both variables are of the same order (e.g. $\alpha$ vs $C_{D}$ ). This treatment requires a two variable regression polynomial fit which is subject to scaling concerns and requires different statistical analysis tools then described by Wahls et al. ${ }^{19}$

The repeatability method that is described utilizes a sample problem of actual data from the cryogenic portion of the NTF test. The chosen example utilizes $\alpha$ for the x-data and $C_{D}$ (stability-axis) for the y-data. Using the $\mathrm{x}$-data from the runs, an arithmetic mean is calculated and designated as the nominal reference $\mathrm{x}$-data values ( $\mathrm{x}$-nom in Table 3). It is worthwhile to note that this choice of nominal $\mathrm{x}$-data values is arbitrary as the existing NTF code allows for user defined nominal x-data values. The computed set of common x-nom values are then used to determine the residuals for all repeat runs. This is accomplished by utilizing a linear curve fit including extrapolation ${ }^{\mathrm{a}}$. In particular, the $\mathrm{x}$-data and $\mathrm{y}$-data for each individual run is used to perform y-data interpolation (y-int in Table 3) at all of the designated x-nom points. The resulting $\mathrm{y}$-int values are averaged at each individual $\mathrm{x}$-nom point to produce nominal y values (y-nom in Table 3). Now at this stage, we have obtained a reference run (x-nom,y-nom) and interpolated data for each run (x-nom,y-interp). The residuals for each polar point (y-resid in Table 3) for each run are computed as:

$$
\delta Y_{i, j}=\left(Y_{i, j}-\bar{Y}_{j}\right) \quad i=1,2, \ldots, M \quad j=1,2, \ldots, N
$$

where $M$ is the total number of repeat runs while $N$ is the total number of points per polar run. Next the sampled standard deviation for each polar run $\left(s_{i}\right.$ in Table 3$)$ is found using:

\footnotetext{
a Linear extrapolation performed using Matlab(c) command "interp1".
} 


$$
s_{i}=\left[\frac{\sum_{j=1}^{N}\left(Y_{i, j}-\bar{Y}_{j}\right)^{2}}{N-1}\right]^{\frac{1}{2}}=\left[\frac{\sum_{j=1}^{N}\left(\delta Y_{i, j}\right)^{2}}{N-1}\right]^{\frac{1}{2}} \quad i=1,2, \ldots, M \quad j=1,2, \ldots, N
$$

where $M$ and $N$ are defined as stated above. Finally, the data repeatability ( $2 \sigma_{\text {mean }}$ in Table 3$)$ is computed using:

$$
2 \sigma_{\text {mean }}=\frac{2 \sum_{i=1}^{M} s_{i}}{M} \quad i=1,2, \ldots, M
$$

The $2 \sigma_{\text {mean }}$ value is then quoted as the data repeatability. Table 3 shows the basic computations for the repeat runs of the NTF CRM Test 197 Series 17.

Some discussion of limitations of this method are warranted. It is important that the data runs used to compute the mean reference run must each have the same number of polar points taken at very similar $\mathrm{x}$-data locations (e.g. $\alpha$ ). This requirement is necessary since averaging is used to compute the $\mathrm{x}$-nom values and the associated y-nom values. Since this method utilizes linear interpolation (at the computed x-nom values) on functions which are only very locally nearly linear, it is important to utilize data points which are taken at nearly the same value for each x-data point as the reference run. Failure to do so will result in utilizing a linear fit for a very non-linear function which will yield substantial inaccuracies. Also, this method suffers (again due to linear interpolation) for regions where the curvature of the reference function is high (e.g. minimum drag for $\alpha$ versus $C_{D}$ ).

Despite any shortcomings, the method utilized in this paper was deemed sufficiently accurate to enable comparison of data quality performance both with and without active damping. The results computed in Table 3 are plotted graphically in Fig. 3.

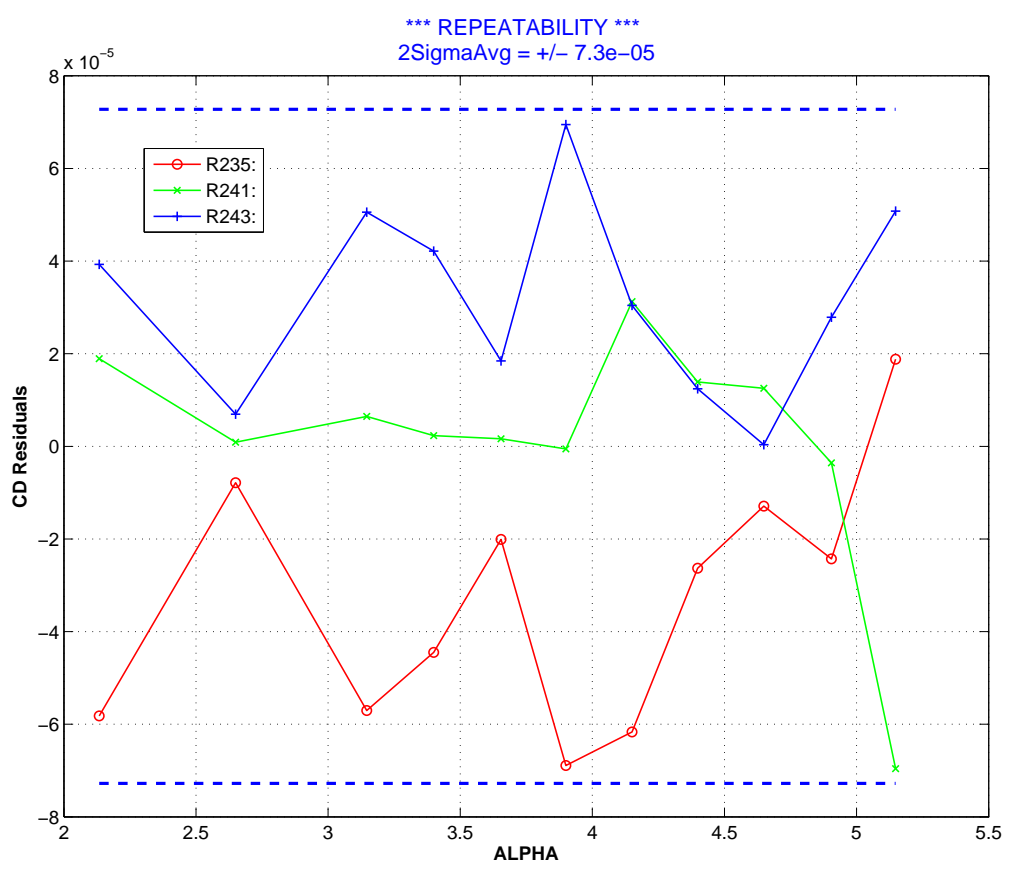

Figure 3. NTF test 197: runs 235,241,243;Mach 0.7, Re 31.9 million/foot, $-182^{\circ}$ F, config: wing/body, no trip dots, active damping off. 


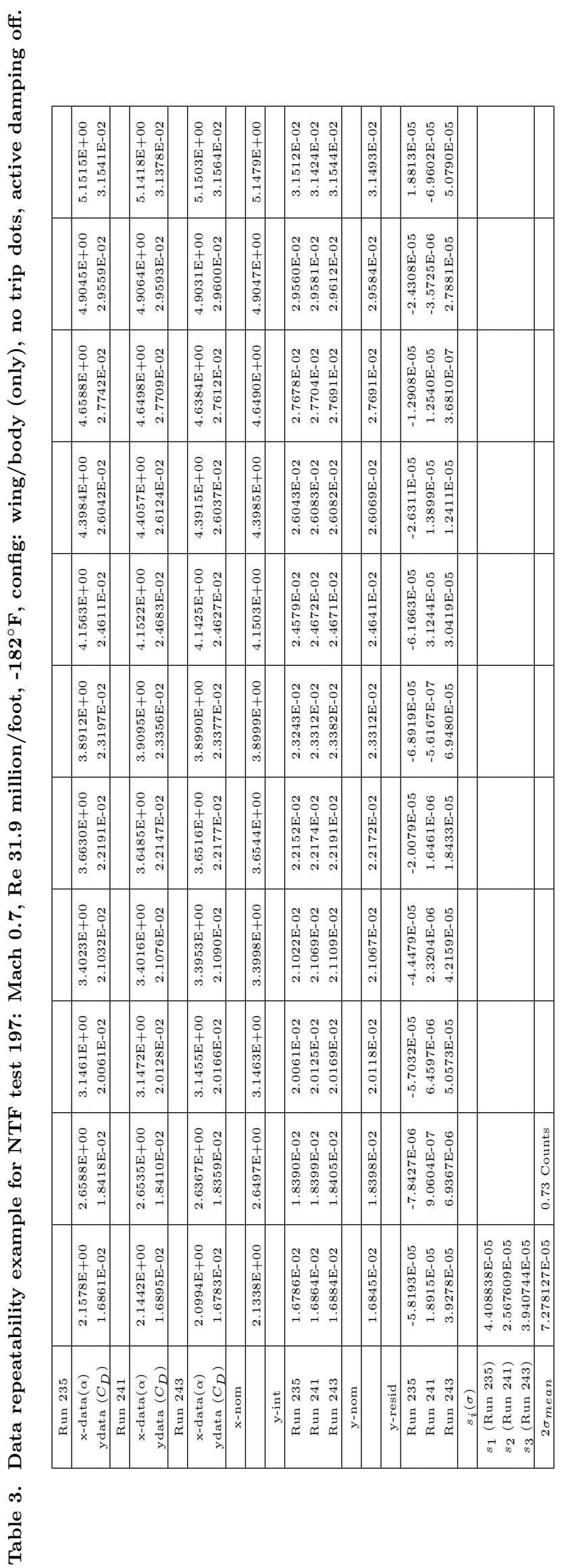

9 of 23 


\section{B. Common Research Model Repeatability Plots}

The enclosed plots document many of the repeat runs and results from NTF Tests 197, 210 and 11' TWT Test 11-0216, while the summarized results have been presented in tabular form previously. These plots represent all test conditions for which repeat data where taken to enable active damping comparisons but don't represent a complete analysis of all repeat runs taken during the tests. To ensure similar computations, the results shown for NTF Test 197 and 11' TWT Test 11-0216 utilize the same number of points per polar (13) which would otherwise have a non-trivial effect on the comparison of standard deviations. However, the repeat data taken during NTF Test 210 (all runs with active damping off) have significantly fewer points per polar (7 versus 13) and should be expected to yield slightly increased standard deviations in practice.
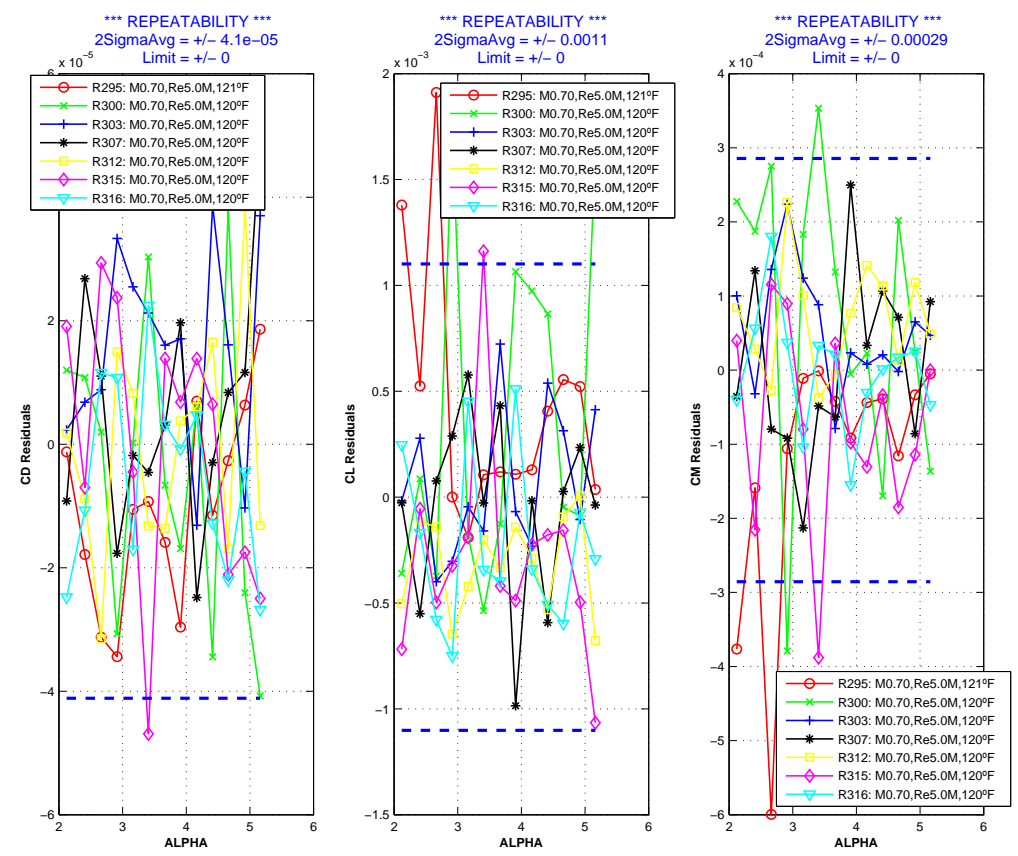

Figure 4. NTF test 197: Mach 0.7, Re 8.06 million/foot, $120^{\circ} \mathrm{F}$, config: wing/body $0^{\circ}$ tails, trip dots on, active damping on, within series repeatability. 

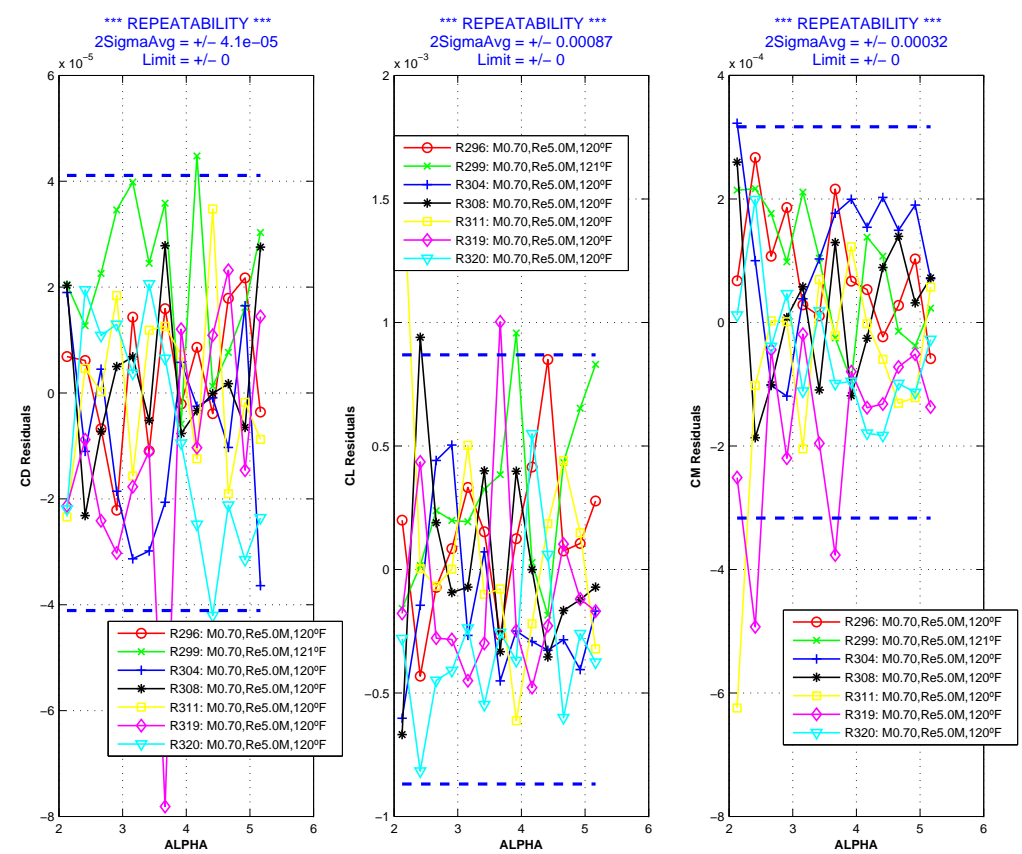

Figure 5. NTF test 197: Mach 0.7, Re 8.06 million/foot, $120^{\circ} \mathrm{F}$, config: wing $/$ body $0^{\circ}$ tails, trip dots on, active damping off, within series repeatability.
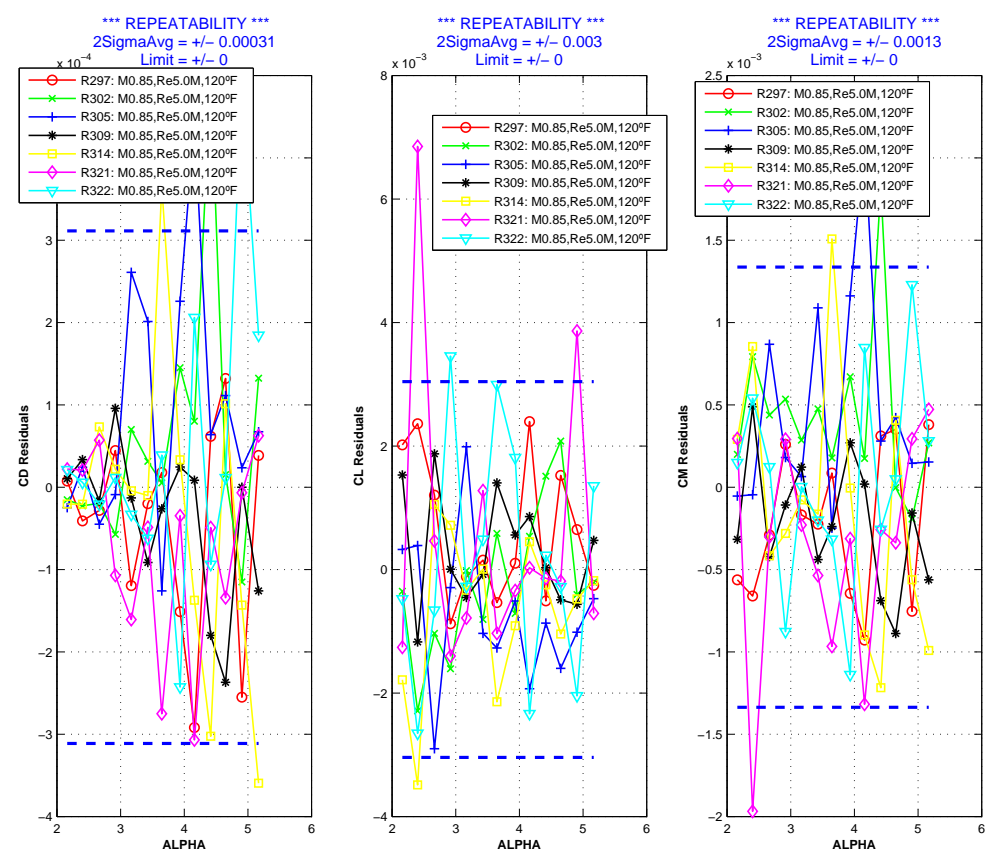

Figure 6. NTF test 197: Mach 0.85 , Re 8.06 million/foot, $120^{\circ} \mathrm{F}$, config: wing/body $0^{\circ}$ tails, trip dots on, active damping on, within series repeatability. 

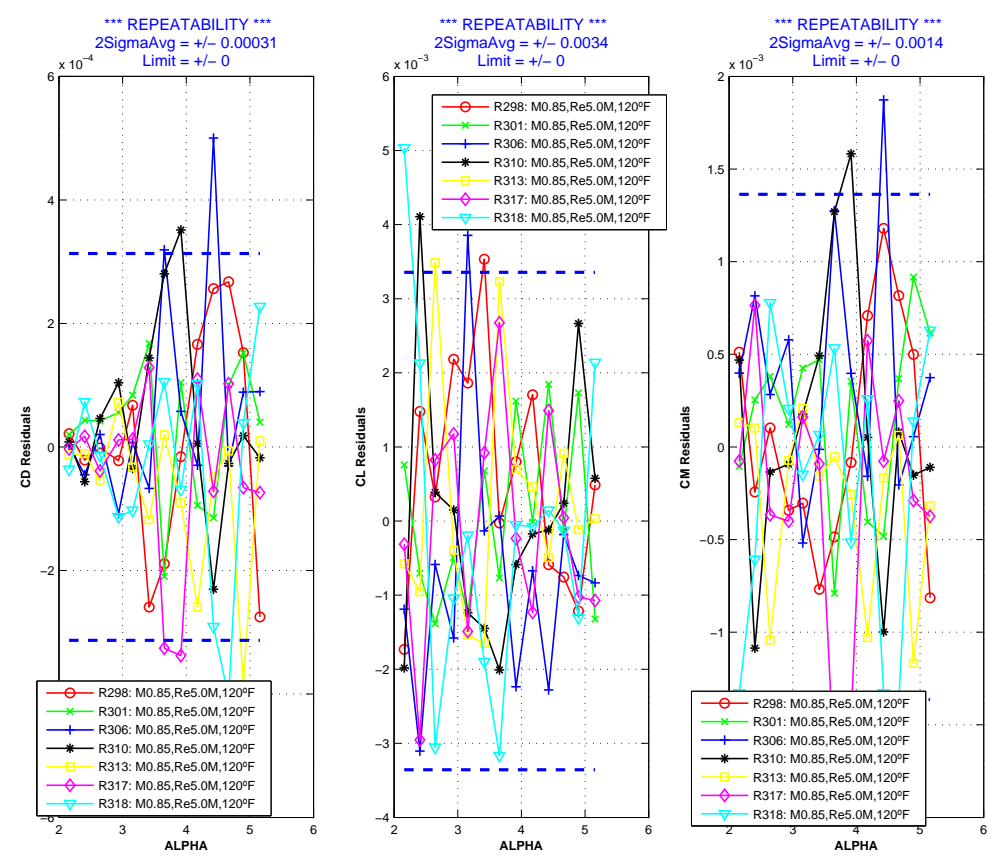

Figure 7. NTF test 197: Mach 0.85, Re 8.06 million/foot, $120^{\circ} \mathrm{F}$, config: wing/body $0^{\circ}$ tails, trip dots on, active damping off, within series repeatability.
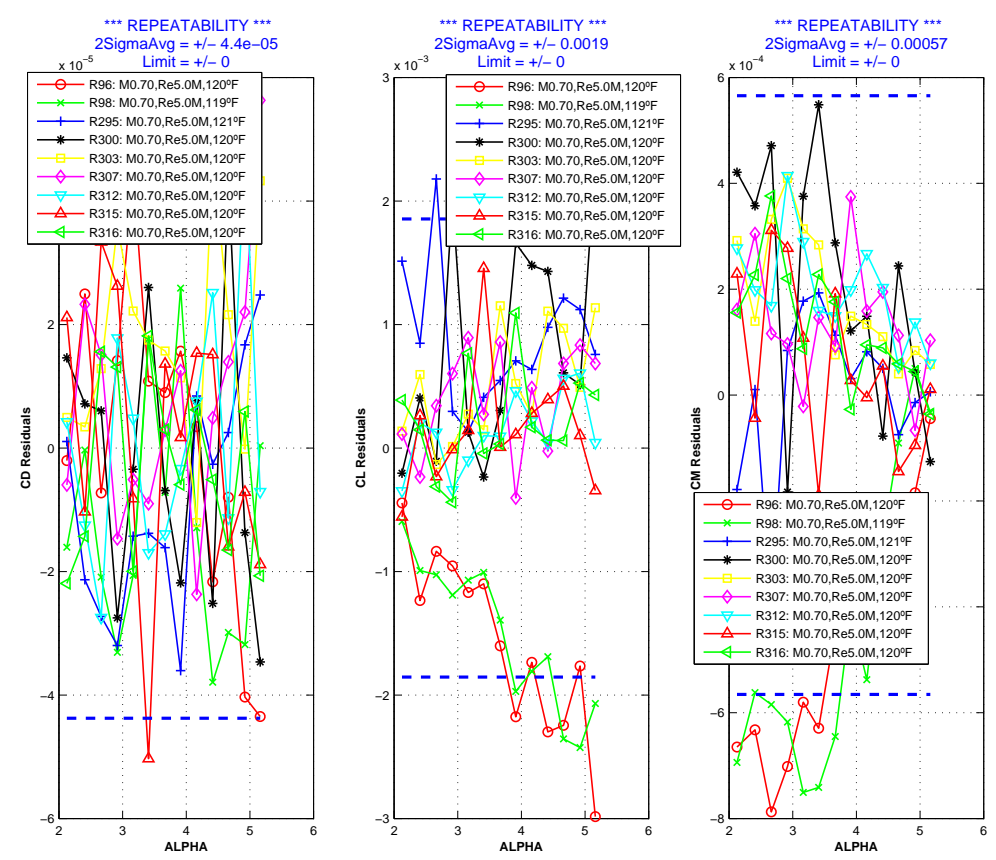

Figure 8. NTF test 197: Mach 0.7, Re 8.06 million/foot, $120^{\circ} \mathrm{F}$, config: wing/body $0^{\circ}$ tails, trip dots on, active damping on, within test repeatability. 

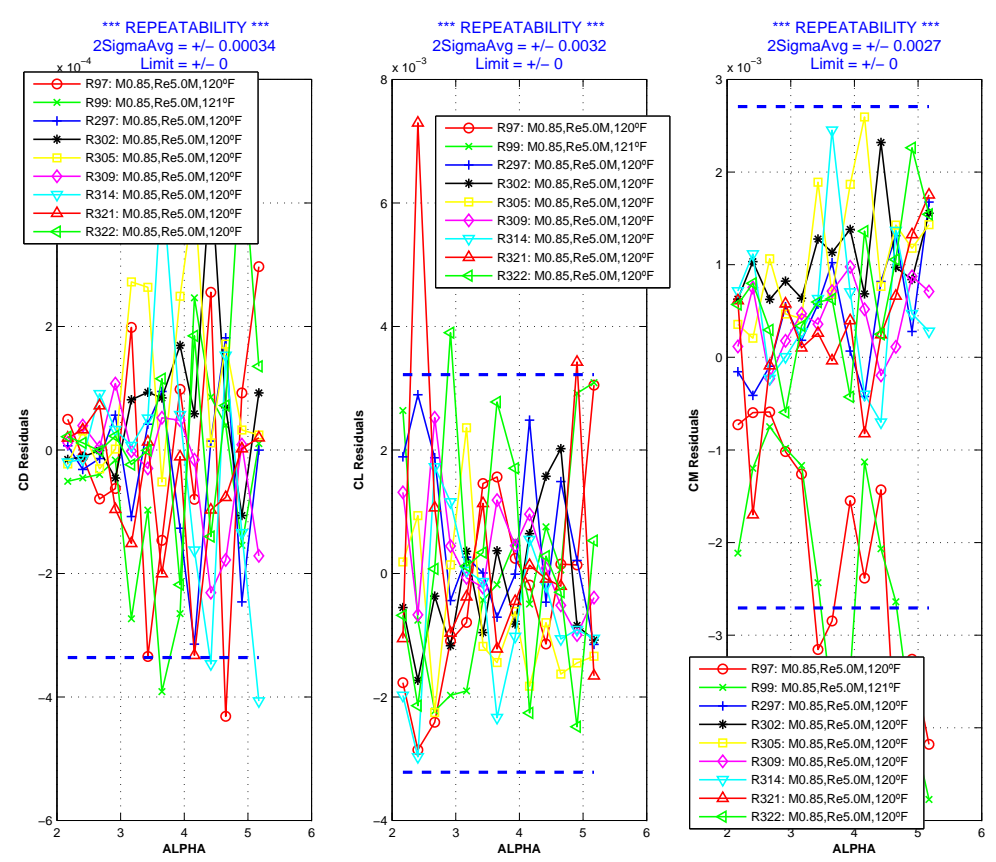

Figure 9. NTF test 197: Mach 0.85, Re 8.06 million/foot, $120^{\circ} \mathrm{F}$, config: wing/body $0^{\circ}$ tails, trip dots on, active damping on, within test repeatability.
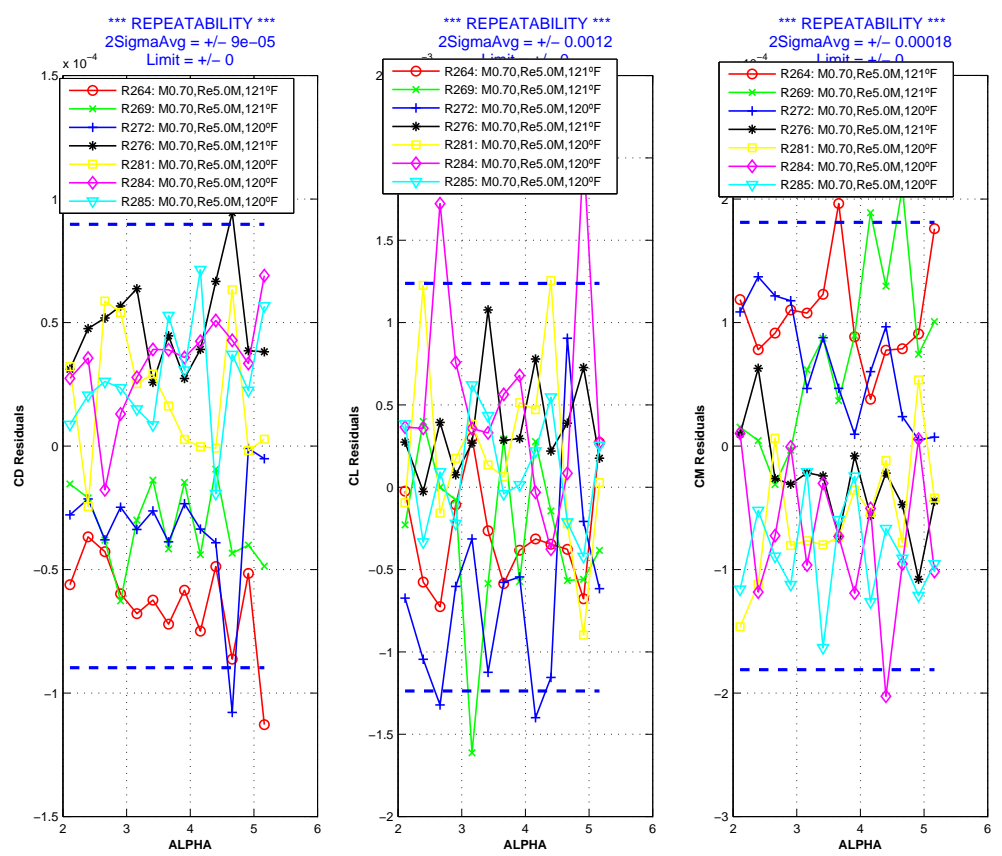

Figure 10. NTF test 197: Mach 0.7, Re 8.06 million/foot, $120^{\circ} \mathrm{F}$, config: wing/body, trip dots on, active damping on, within series repeatability. 

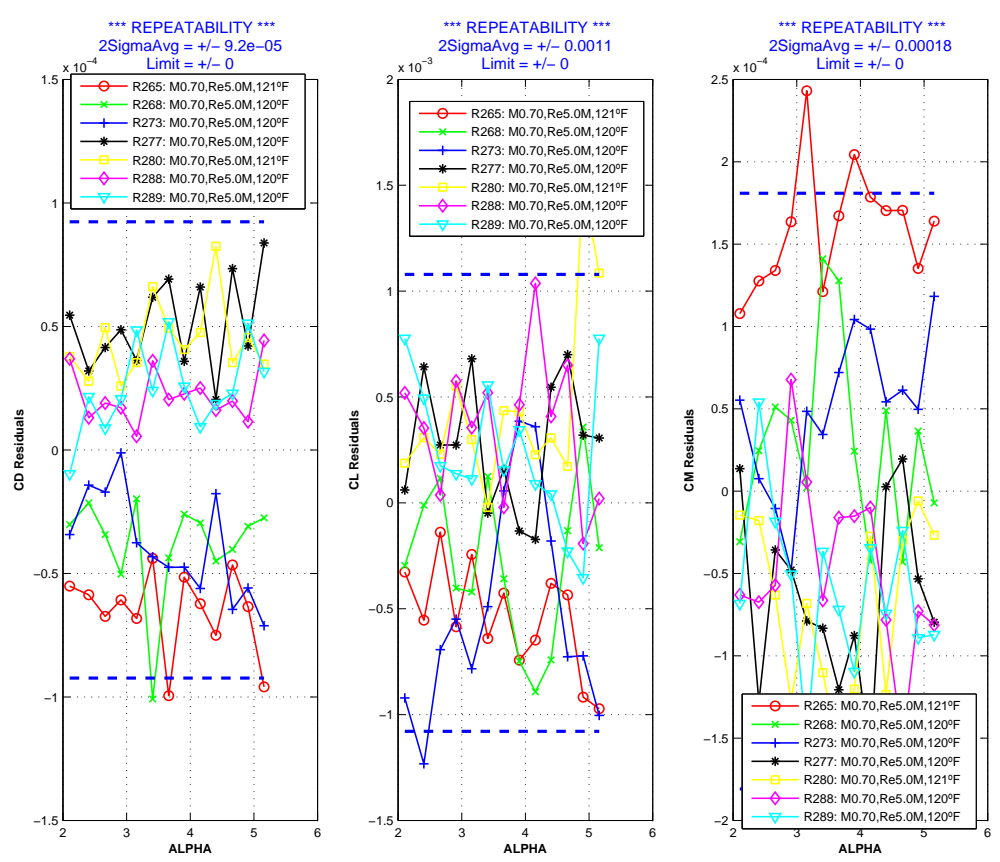

Figure 11. NTF test 197: Mach 0.7, Re 8.06 million/foot, $120^{\circ}$ F, config: wing/body, trip dots on, active damping off, within series repeatability.
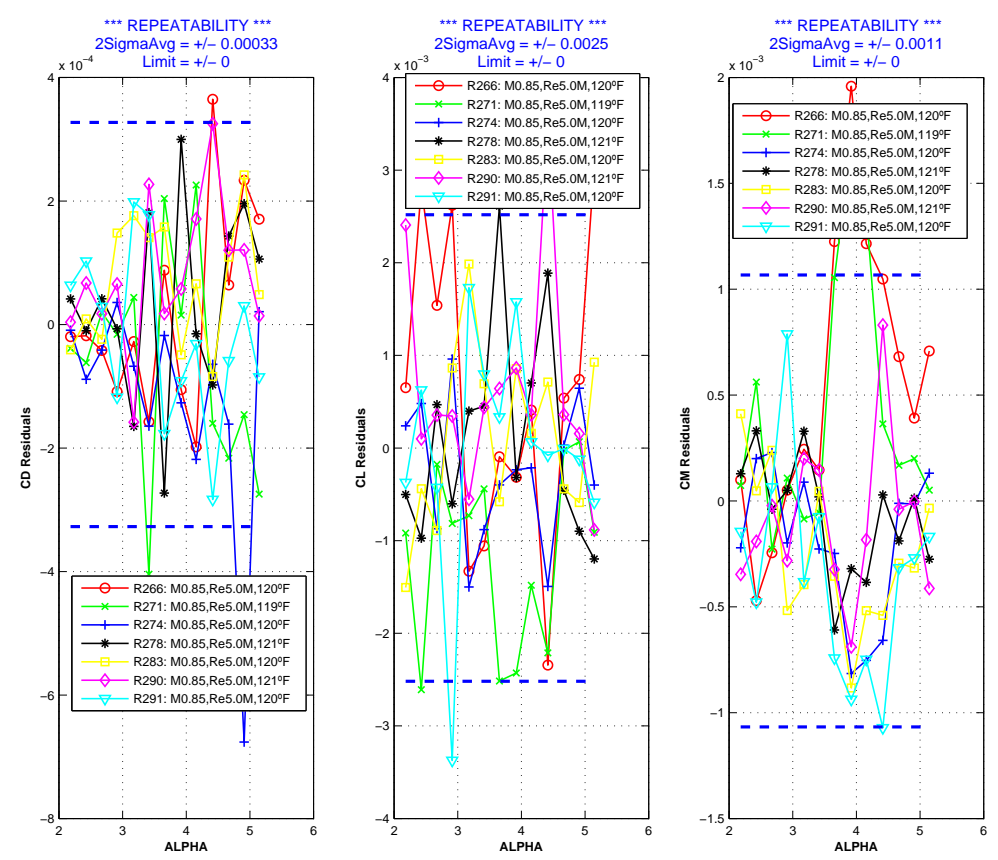

Figure 12. NTF test 197: Mach 0.85, Re 8.06 million/foot, $120^{\circ}$ F, config: wing/body, trip dots on, active damping on, within series repeatability. 

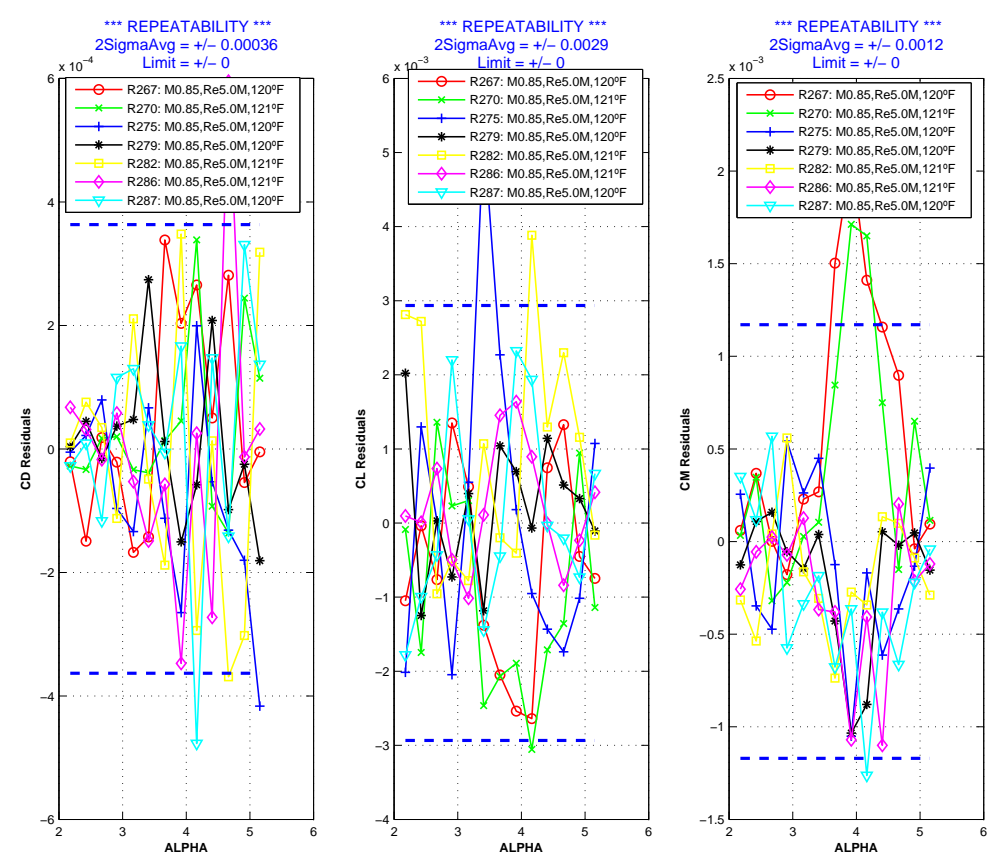

Figure 13. NTF test 197: Mach 0.85, Re 8.06 million/foot, $120^{\circ}$ F, config: wing/body, trip dots on, active damping off, within series repeatability.
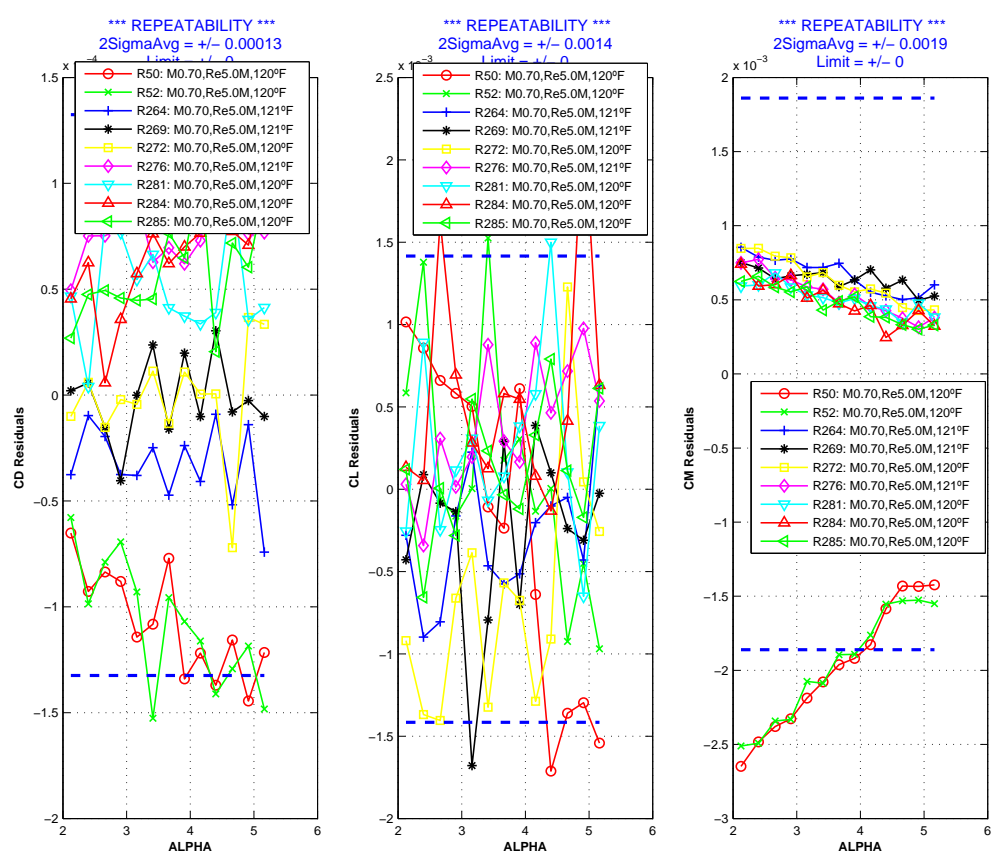

Figure 14. NTF test 197: Mach 0.7, Re 8.06 million/foot, $120^{\circ} \mathrm{F}$, config: wing/body, trip dots on, active damping on, within test repeatability. 

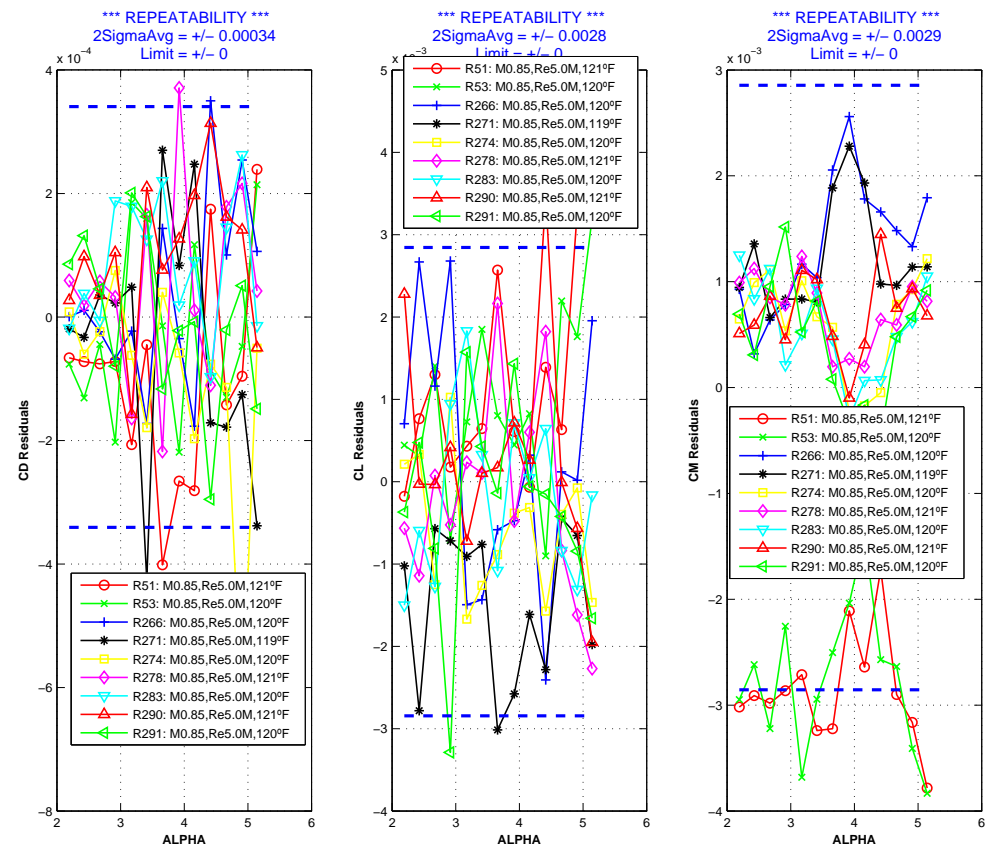

Figure 15. NTF test 197: Mach 0.85 , Re 8.06 million/foot, $120^{\circ}$ F, config: wing/body, trip dots on, active damping on, within test repeatability. 

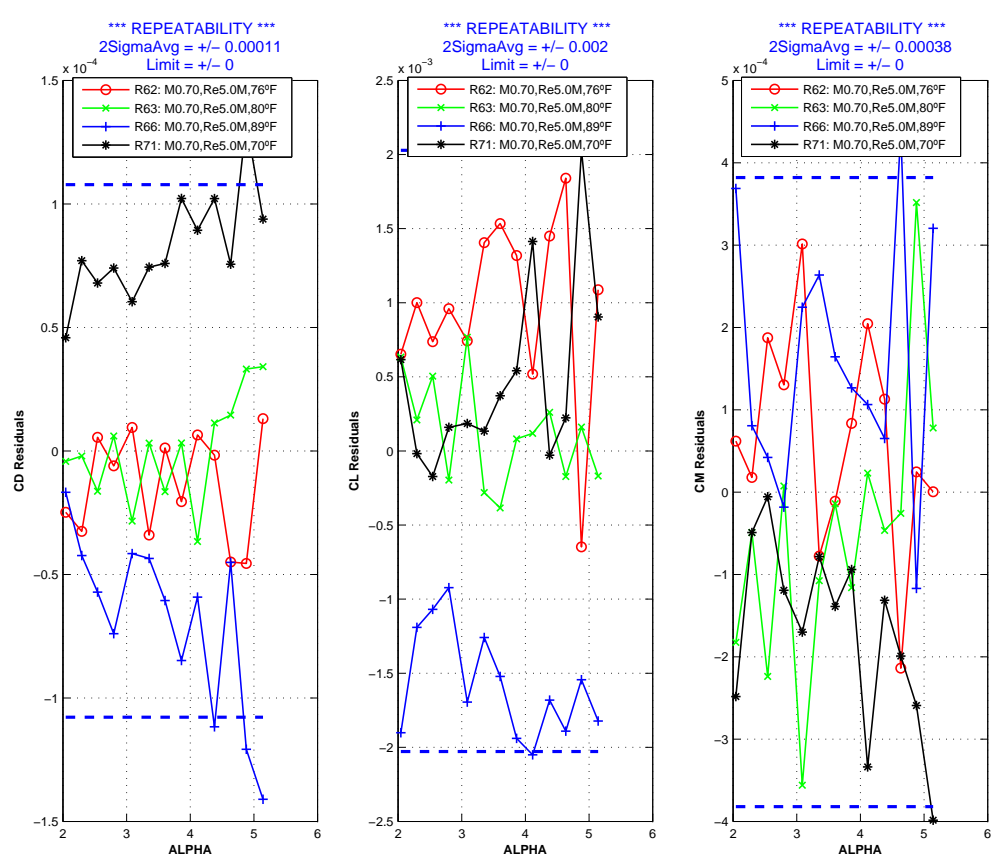

Figure 16. 11FT TWT test 11-0216: Mach 0.7, Re 8.06 million/foot, config: wing/body $0^{\circ}$ tails, trip dots on, active damping on, within series repeatability.
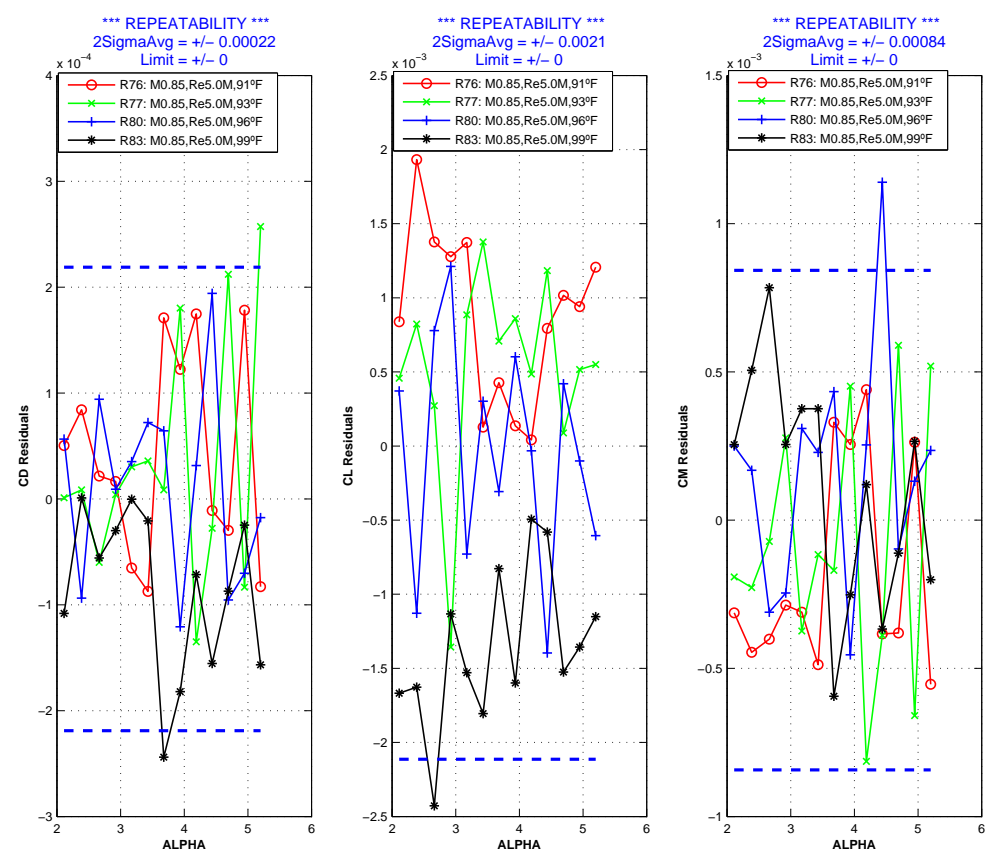

Figure 17. 11FT TWT test 11-0216: Mach 0.85, Re 8.06 million/foot, config: wing/body $0^{\circ}$ tails, trip dots on, active damping on, within series repeatability. 

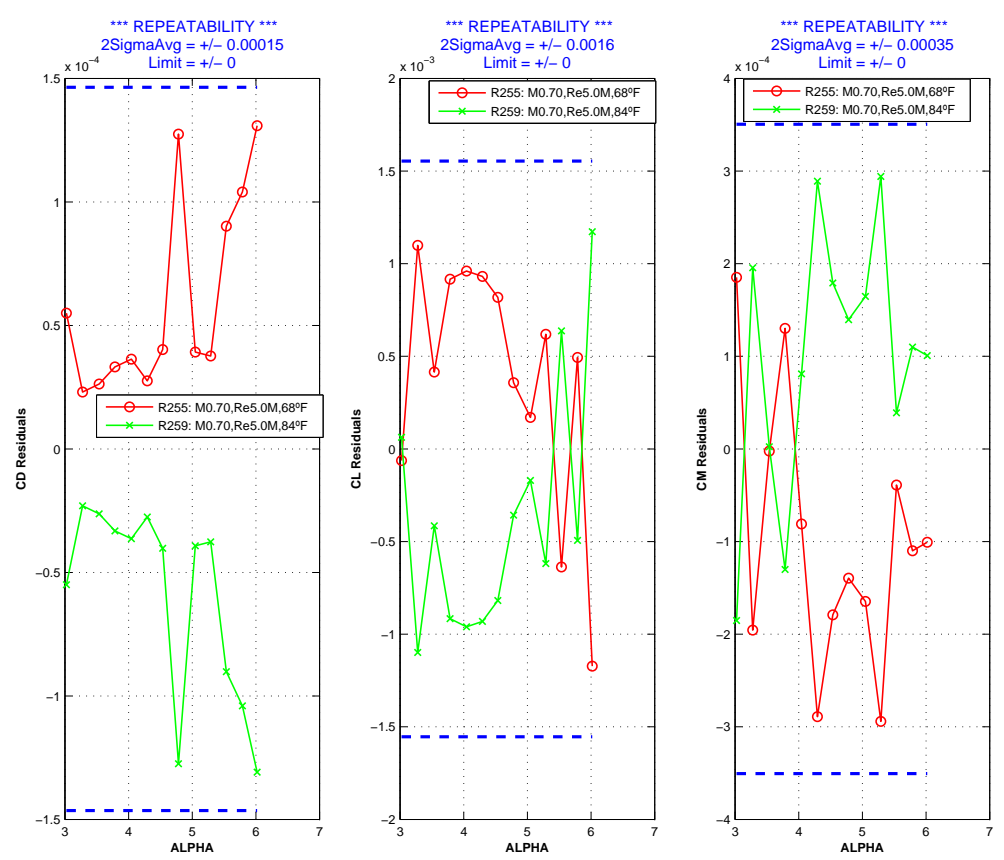

Figure 18. 11FT TWT test 11-0216: Mach 0.7, Re 8.06 million/foot, config: wing/body $0^{\circ}$ tails, trip dots on, active damping off, conditional sampling on, within series repeatability.
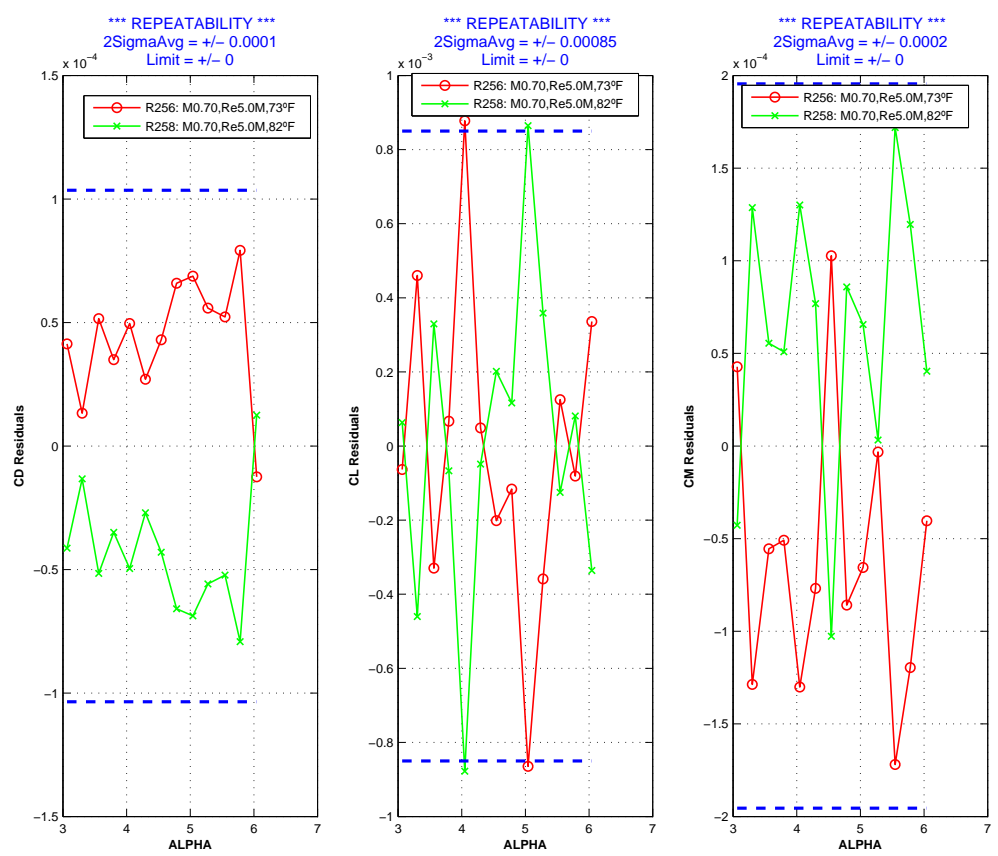

Figure 19. 11FT TWT test 11-0216: Mach 0.7, Re 8.06 million/foot, config: wing/body $0^{\circ}$ tails, trip dots on, active damping off, conditional sampling off, within series repeatability. 

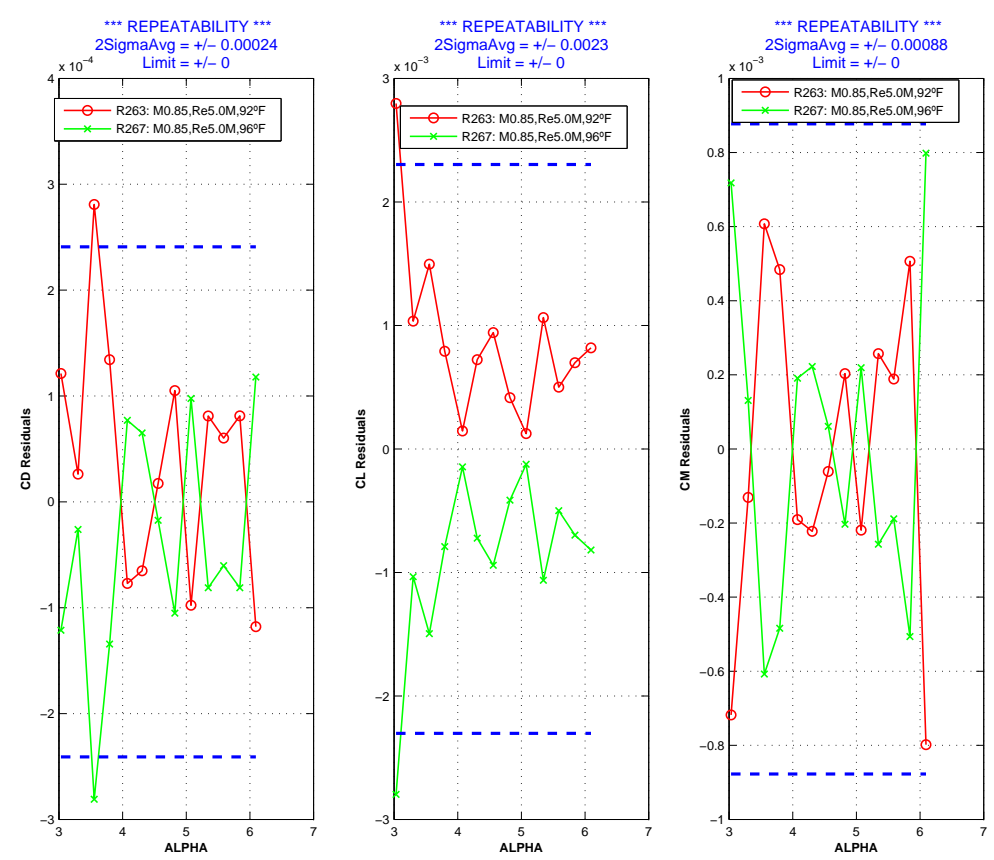

Figure 20. 11FT TWT test 11-0216: Mach 0.85, Re 8.06 million/foot, config: wing/body $0^{\circ}$ tails, trip dots on, active damping off, conditional sampling on, within series repeatability.
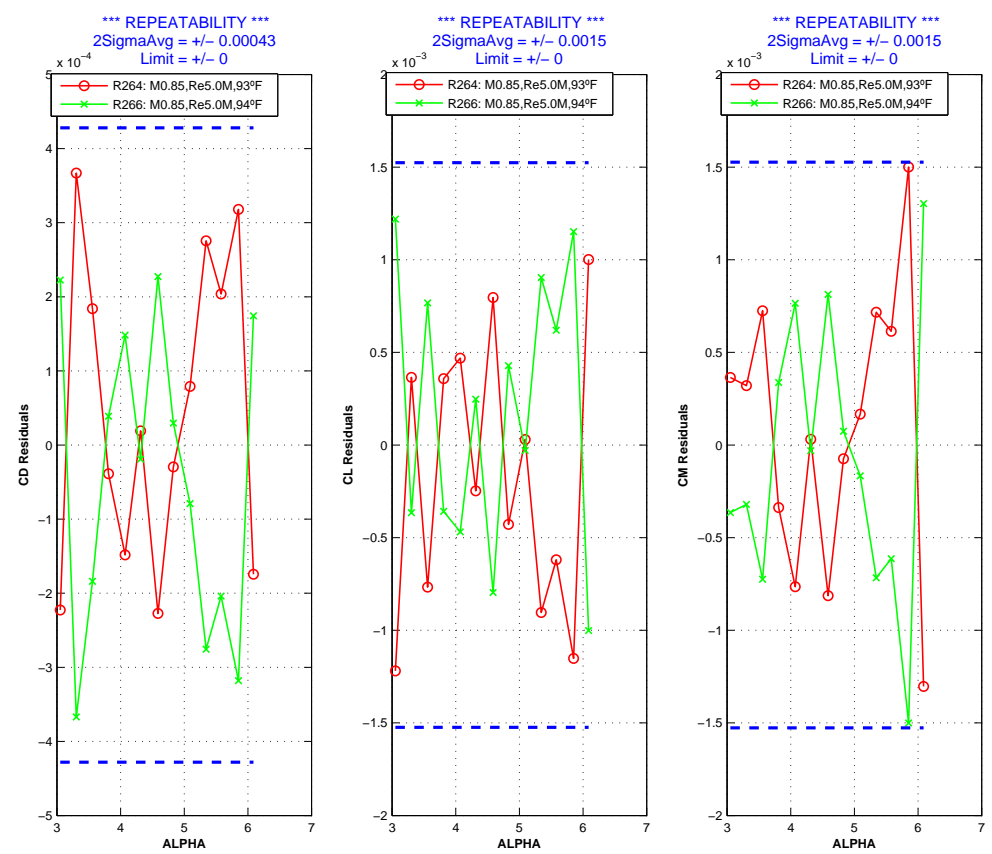

Figure 21. 11FT TWT test 11-0216: Mach 0.85, Re 8.06 million/foot, config: wing/body $0^{\circ}$ tails, trip dots on, active damping off, conditional sampling off, within series repeatability. 

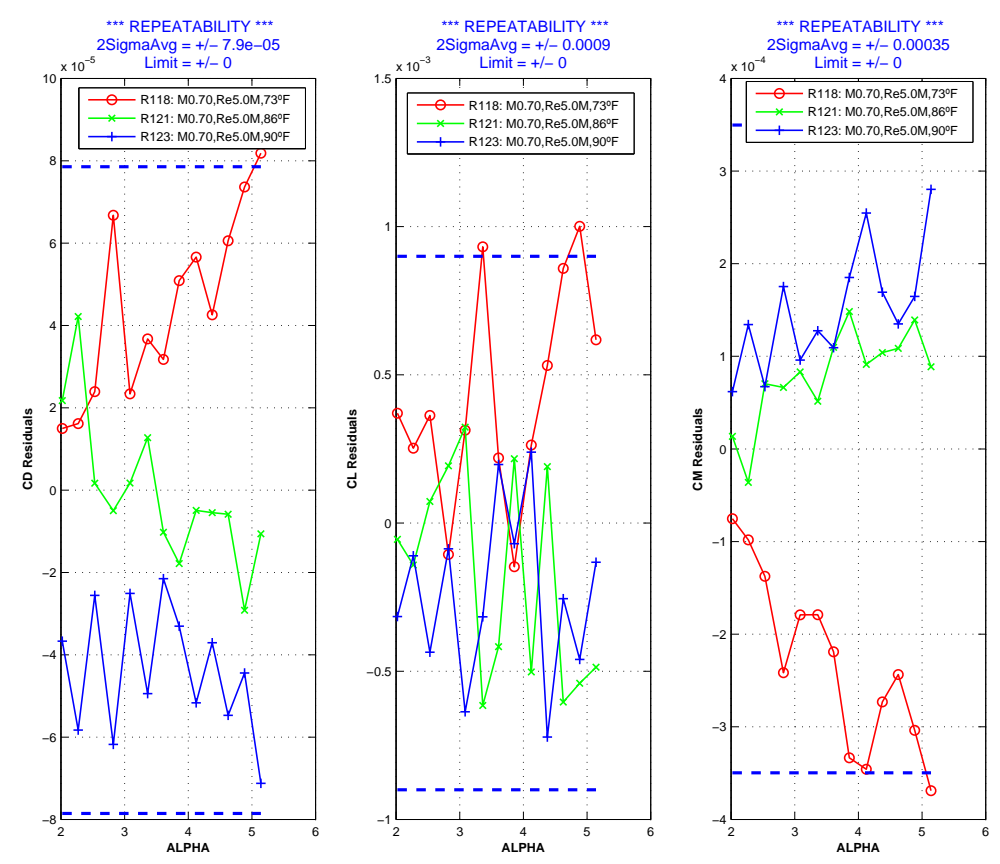

Figure 22. 11FT TWT test 11-0216: Mach 0.7, Re 8.06 million/foot, config: wing/body, trip dots on, active damping on, within series repeatability.
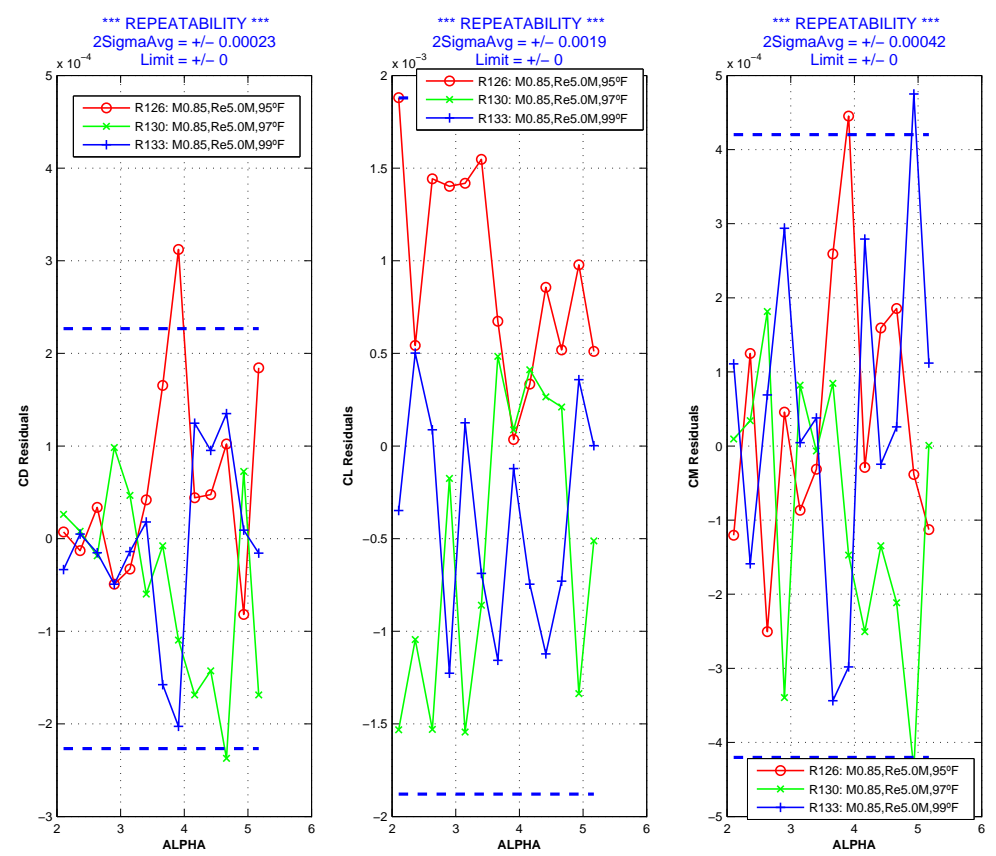

Figure 23. 11FT TWT test 11-0216: Mach 0.85, Re 8.06 million/foot, config: wing/body, trip dots on, active damping on, within series repeatability. 

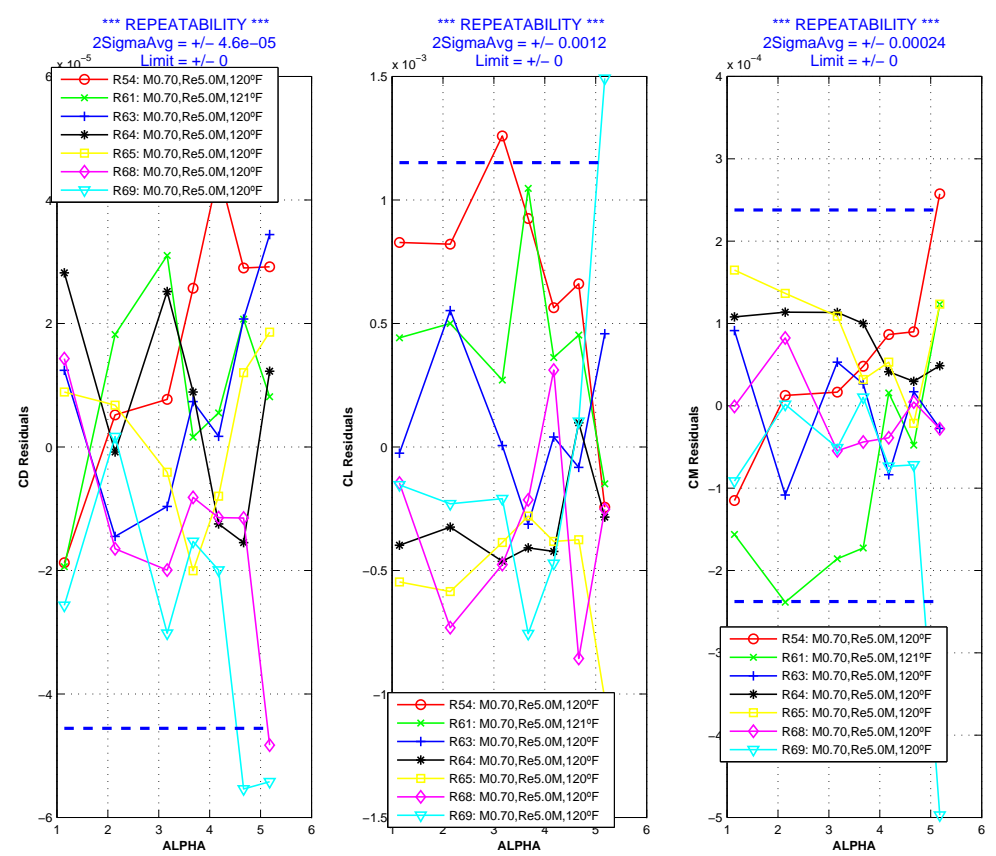

Figure 24. NTF test 210: Mach 0.7, Re 8.06 million/foot, $120^{\circ} \mathrm{F}$, config: wing/body $0^{\circ}$ tails, trip dots on, active damping off, within series repeatability.
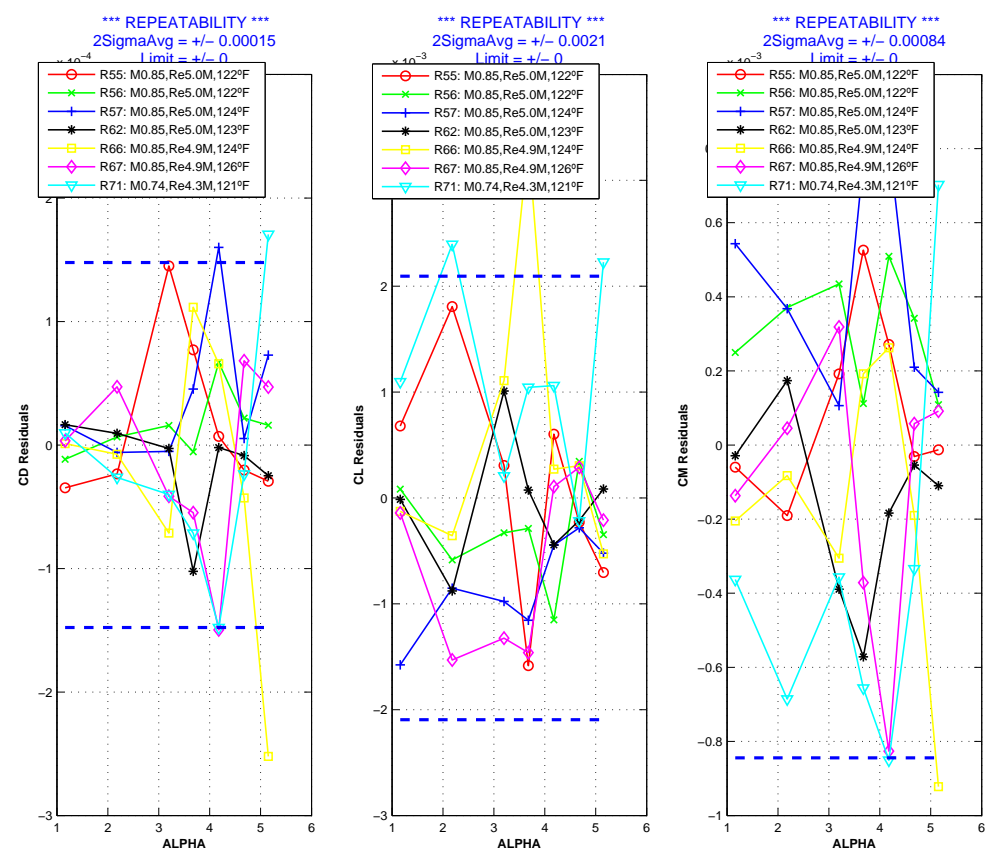

Figure 25. NTF test 210: Mach 0.85 , Re 8.06 million/foot, $120^{\circ}$ F, config: wing/body $0^{\circ}$ tails, trip dots on, active damping off, within series repeatability. 


\section{Acknowledgments}

The authors would like to gratefully acknowledge the dedication and commitment to excellence of the NTF test team (also to include David Chan), and the 11' TWT test team as well as the superb support of the Vigyan Inc. personnel David Butler and Wade Saltzgiver. Additionally, the authors acknowledge the encouragement and financial support from Melissa Rivers. Finally, special appreciation is given to Albert (Bruce) Graham and Mathew Asay at the NTF whose support and data reduction software development has been critical in the unique analysis of NTF Test 210 data sampling evaluations. 


\section{References}

${ }^{1}$ Billingsley James P., "Sting Dynamics of Wind Tunnel Models," AEDC -TR-76-41, June 1975.

${ }^{2}$ Kilgore, W.A., Balakrishna, S., and Butler, D.H., "Reduction of Tunnel Dynamics at the National Transonic Facility," AIAA Paper 2001-1162.

${ }^{3}$ Weiss J., "Model Vibrations and Inertial Bias Measurement in a Transonic Wind Tunnel Test," AIAA Paper 2008-4033, June 2008.

${ }^{4}$ Young, C., Popernack T. and Gloss B., "National Transonic Facility Model and Model Support Vibration Problems," AIAA Paper 1990-1416, June 1990.

${ }^{5}$ Edwards, J. W., "National Transonic Facility Model and Tunnel Vibrations," Journal of Aircraft, Vol. 46, No. 1, JanuaryFebruary 2009.

${ }^{6}$ Whitlow, W., Bennett, R. and Strganac, T., "Analysis of Vibrations of the National Transonic Facility Model Support System Using a 3-D Aeroelastic Code," AIAA Paper 1989-2207, July 1989.

${ }^{7}$ Parker, P. A., "Wind Tunnel Model System Dynamic Analysis and Simulation with Application to Model System Vibration Suppression," Masters Thesis, Department of Applied Physics and Computer Science, Christopher Newport University, Newport News, VA, 2000.

${ }^{8}$ Buehrle, R. D., "System Dynamic Analysis of a Wind Tunnel Model with Applications to Improve Aerodynamic Data Quality," Doctoral Dissertation, Department of Mechanical, Industrial, and Nuclear Engineering of the College of Engineering, University of Cincinnati, Cincinnati, OH, 1997.

${ }^{9}$ Crawford B. and Finley T., "Improved Correction System for Vibration Sensitive Inertial Angle of Attack Measurement Devices," AIAA Paper 2000-0415, January 2000.

${ }^{10}$ Crawford B. and Finley T., "Results From a Sting Whip Correction Verification Test at the Langley 16-Foot Transonic Tunnel," AIAA Paper 2002-0879, January 2002.

${ }^{11}$ Fehren,H., Gnauert U., and Wimmel R., "Validation Testing with the Active Damping System in the European Transonic Windtunnel," AIAA Paper 2001-0610, January 2001.

${ }^{12}$ Balakrishna S., Houlden Heather, Butler D.H., and White Richard, "Development of a Wind Tunnel Active Vibration Reduction System", AIAA Paper 2007-961 January, 2007.

${ }^{13}$ Laster Marion L., "Aerodynamic Data Accuracy and Quality: Requirements and Capabilities in Wind Tunnel Testing," Technical Evaluation Report on the Fluid Dynamics Panel Symposium, July 1988.

${ }^{14}$ Fuijkschot, P. H., "Looking for the Last Dragcount- Model Vibrations Vs. Drag Accuracy," 1st International Symposium on Strain Gauge Balances, Paper AC-2, March 1999

${ }^{15}$ Vassberg, J., Deehan, M., Rivers, M., and Wahls, R., "Development of a Common Research Model for Applied CFD Validation Studies," AIAA Paper 2008-6919, August 2008.

${ }^{16}$ Balakrishna, S., and Acheson, M., "Analysis of NASA Common Research Model Dynamic Data," 49th AIAA Aerospace Sciences Meeting, American Institute of Aeronautics and Astronautics, Reston, VA (submitted for publication)

${ }^{17}$ Balakrishna, S., Butler, D., Acheson, M., and White, R., "Design \& Performance of an Active Sting Damper for the NASA Common Research Model," 49th AIAA Aerospace Sciences Meeting, American Institute of Aeronautics and Astronautics, Reston, VA (submitted for publication)

${ }^{18}$ Schimanski, D., and Hefer, G., "Recent Aspects of High Reynolds Number Data Quality and Capabilities at the European Transonic WindTunnel," AIAA Paper 2000-0292, January, 2000.

${ }^{19}$ Wahls, R., Adcock, J., Witkowski, D., and Wright, F., "A Longitudinal Aerodynamic Data Repeatability Study for a Commercial Transport Model Test in the National Transonic Facility,” NASA Technical Paper 3522, 1995. 